\title{
Heart Failure with Preserved Ejection Fraction and 30-Day Readmission
}

\author{
Manjari Rani Regmi, MD; Mukul Bhattarai MD, MPH, FACP; Priyanka Parajuli, MD; \\ Odalys Estefania Lara Garcia, MD; Nitin Tandan, MD; Nicolas Ferry, MD, Capt USAF; \\ Asad Cheema, MD; Youssef Chami, MD; and Robert Robinson, MD, MS, FACP
}

\begin{abstract}
Objective: Several studies identify heart failure (HF) as a potential risk for hospital readmission; however, studies on predictability of heart failure readmission is limited.The objective of this work was to investigate whether a specific type of heart failure (HFpEF or HFrEF) has a higher association to the rate of 30-day hospital readmission and compare their predictability with the two risk scores: HOSPITAL score and LACE index.
\end{abstract}

Design: Retrospective study from single academic center.

Methods: Sample size included adult patients from an academic hospital in a two-year period (2015 2017). Exclusion criteria included death, transfer to another hospital, and unadvised leave from hospital. Baseline characteristics, diagnosis-related group, and ICD diagnosis codes were obtained.Variables affecting HOSPITAL score and LACE index and types of heart failure present were also extracted. Qualitative variables were compared using Pearson chi $^{2}$ or Fisher's exact test (reported as frequency) and quantitative variables using non-parametric Mann-Whitney $U$ test (reported as mean \pm standard deviation). Variables from univariate analysis with $P$ values of 0.05 or less were further analyzed using multivariate logistic regression. Odds ratio was used to measure potential risk.

Results: The sample size of adult patients in the study period was 1,916. All eligible cohort of patients who were readmitted were analyzed. Cumulative score indicators of HOSPITAL Score, LACE index (including the Charlson Comorbidity Index) predicted 30-day readmissions with $P$ values of $<0.00$ I. The $P$ value of HFpEF was found to be significant in the readmitted group $(P<0.00 \mathrm{I})$ compared to HFrEF $(P=$ 0.14I). Multivariate logistic regression further demonstrated the association of HFpEF with higher risk of readmission with odds ratio of $\mathrm{I} .77(95 \% \mathrm{Cl}$ : I.25 - 2.50) and $P$ value of $0.00 \mathrm{I}$.

Conclusions: Our data from an academic tertiary care center supports HFpEF as an independent risk factor for readmission. Multidisciplinary management of HFpEF may be an important target for interventions to reduce hospital readmissions.

Keywords: HFpEF; HFrEF; 30-day readmission; Heart Failure; LACE index; HOSPITAL score

A pproximately 5 million Americans are currently diagnosed with Congestive Heart Failure (CHF) thereby making CHF a common disease entity. Over 550,000 new cases are diagnosed each year, and it is the most common diagnosis in patients over the age of $65 .{ }^{1} \mathrm{An}$ estimated \$20-30 billion is the associated annual cost of treatment of heart failure each year., ${ }^{2,3}$
Heart failure is a clinical syndrome defined as impaired cardiac contractility and ventricular dilatation. There are two types of heart failure: heart failure with reduced ejection fraction (HFrEF) and heart failure with preserved ejection fraction (HFpEF). Heart failure continues to be a primary reason for hospitalization in patients older than 65 years and an issue to health care systems. ${ }^{4}$ In addition to the initial
Corresponding Author: Manjari Rani Regmi, MD; 70I North First Street, Springfield, IL 6278I, Tel: (470) 265-1046, Fax: (279) 20I-6023, Email:mregmi8I@siumed.edu; manjariregmi08.mr@gmail.com
Received: August 5, 2019

Revised: February 19, 2020

Accepted: March I3, 2020

doi: $|0.3| 2|/ \mathrm{cmr} .2020 .152|$ 
admission cases, heart failure remains the leading cause of 30-day hospital readmissions. ${ }^{4}$ National data suggests that heart failure readmission rates have steadily increased over the last decade. ${ }^{5}$ Patients admitted to the hospital for heart failure are prone to readmission risk after their treatment, with some studies finding up to $90 \%$ readmission rate within 90 days. ${ }^{6}$ According to the Centers for Medicaid Services, amongst patients initially hospitalized for heart failure, $25 \%$ were readmitted within 30 days and 35\% among them were readmitted because of heart-failure.

Health care providers as well as policymakers and hospital administrators are actively seeking updated and more effective scoring systems to predict hospital readmissions to help reduce the extremely high rate and costs associated with readmissions of the patient population. Researchers are constantly working towards identifying different variables to develop new scores or further add to the existing scores in an attempt to provide scoring systems that have higher readmission predictability and specificity. ${ }^{8}$ Some of these scores include HOSPITAL score, and LACE index. HOSPITAL score stands for Hemoglobin at discharge, discharge from Oncology service, Sodium level at discharge, Procedure performed, Index of admission Type (elective vs. emergent), number of previous hospital-Admissions, Length of stay, and it was validated by Donzé et $\mathrm{al}^{9}$ in a multicenter study. Similarly, LACE index, that stands for Length of stay, Acuity of admission, Comorbidities, Emergency hospital visits, and it was validated by Van Walraven et $\mathrm{al}^{10}$ to estimate the re-hospitalization risk and showed significant association of higher LACE index score with increased rate of readmissions and mortality.

HOSPITAL scores and LACE indices have been validated and have been known to show correlation with rate of readmissions and mortality. ${ }^{9,10}$ However, a few studies have shown these scores do not reliably predict readmissions or help target the cause of these readmissions. ${ }^{11,12}$ Studies have shown that LACE index is not very effective in accurately predicting 30-day readmissions. ${ }^{11,13,14,15}$ In one retrospective study that included HFpEF patients, both HOSPITAL and LACE index did not show association with increased readmissions. ${ }^{16}$ Very few studies have focused determining the association of the type of heart failure in hospital readmissions. ${ }^{17}$ This provides a motivation to explore the types of heart failure and identify their association with hospital readmission.

The objective of this work was to investigate whether a specific type of heart failure (HFpEF or HFrEF) has a higher association to the rate of 30-day hospital readmission and compare their predictability with the two risk scores: HOSPITAL score and LACE index.

\section{Materials and Methods}

Study Design

All adult medical patients discharged hospitalist service at Memorial Medical Center affiliated with Southern Illinois
University School of Medicine from January 1, 2015 to January 1, 2017 were potentially eligible for this retrospective study. Exclusion criteria included transfer to another acute care hospital, leaving the hospital against medical advice, or death.

\section{Study Setting}

The study setting is a 507-bed not-for-profit tertiary care center. The Hospitalist service is the general internal medicine residency teaching service staffed by board certified and eligible hospitalist faculty. Patients for the hospitalist service are primarily admitted via the hospital emergency department or transferred from other regional hospitals with acute medical issues. Elective hospital admissions are extremely rare for this service. Readmissions at other hospitals were not considered for the study since only data from the study hospital was available. This study obtained the approval from the Institutional Review Board. It was determined that this study does not meet the criteria for research involving human subjects according to 45 CFR 46.101 and 45 CFR 46.102.

\section{Data Collection}

Data on age, gender, Diagnosis Related Group (DRG), International Classification of Disease (ICD) diagnosis codes, medications on discharge, 30-day readmission status, heart failure and its types, variables needed to calculate HOSPITAL, LACE, and Charlson scores were extracted from the electronic health record. The data was de-identified for analysis for all eligible patients. We looked into both cardiac and non-cardiac readmissions within the aforementioned time frame.

\section{Statistical Analysis}

Qualitative variables were compared using Pearson chi $^{2}$ or Fisher's exact test and reported as frequency (\%). Quantitative variables were compared using the non-parametric MannWhitney $U$ test and reported as mean \pm standard deviation. Variables from univariate analysis with a $P$ value of 0.05 or less were further analyzed using multivariate logistic regression in a backwards likelihood ratio method. The HOSPITAL score and LACE index were calculated at the time of discharge. Based on the initial validation study, HOSPITAL scores between 0 and 4 were classified as low risk for readmission $(5 \%)$, between 5 and 6 as intermediate risk $(10 \%)$, and 7 or more as high risk $(20 \%)$. LACE indices ranged from 0 to 19 , with an expected probability for readmission of up to $43.7 \%$ based on the initial validation study of the LACE score. Statistical analyses were performed using SPSS version 22 (SPSS Inc, Chicago, IL, USA). To test the influence of a variable on readmission, $P$ values were calculated for each variable. Two-sided $P$ values $(<0.05)$ were considered significant.

\section{Results}

Out of 1916 patients, the eligible sample size was 1781. Of them, 456 (25\%) were readmitted within 30 days shown in Figure 1. The results shown in Table 1 summarize the statistics of various qualitative and quantitative variables for 
Table1. Baseline characteristics of study population by 30-day readmission (univariate analysis)

\begin{tabular}{|c|c|c|c|}
\hline Baseline characteristics & $\begin{array}{l}\text { Not readmitted within } \\
30 \text { days }(n=1325)\end{array}$ & $\begin{array}{l}\text { Readmitted within } \\
30 \text { days }(n=456)\end{array}$ & $P$ value \\
\hline Age (mean) (SD) & $63(16.0)$ & $64(15.5)$ & 0.144 \\
\hline Female (n) (\%) & $624(47)$ & $216(47)$ & 0.919 \\
\hline Length of stay (mean) (SD) & $7.7(7.1)$ & $8.4(8.8)$ & 0.091 \\
\hline Hospital admissions last year (mean) (SD) & $0.71(0.8)$ & $1.61(1.7)$ & $<0.001$ \\
\hline ED visits in last 6 months (mean) (SD) & $0.39(1.22)$ & $1.21(3.0)$ & $<0.001$ \\
\hline HOSPITAL Score (mean) (SD) & $3.86(1.4)$ & $4.96(1.8)$ & $<0.001$ \\
\hline LACE Index (mean) (SD) & $11.31(2.4)$ & $12.59(3.72)$ & $<0.001$ \\
\hline Charlson Comorbidity Index (mean)(SD) & $4.88(3.27)$ & $6(3.78)$ & $<0.001$ \\
\hline \multicolumn{4}{|l|}{ Medical Comorbidities } \\
\hline Myocardial infarction (n) (\%) & $344(26)$ & $150(33)$ & 0.004 \\
\hline Peripheral artery disease $(n)(\%)$ & $118(9)$ & $45(10)$ & 0.539 \\
\hline Stroke (n) (\%) & $76(6)$ & $26(6)$ & 0.978 \\
\hline Dementia (n) (\%) & $40(3)$ & $9(2)$ & 0.239 \\
\hline Chronic lung disease (n) (\%) & $357(27)$ & $144(32)$ & 0.058 \\
\hline Connective tissue disease $(n)(\%)$ & $28(2)$ & $7(2)$ & 0.443 \\
\hline Peptic ulcer disease (n) (\%) & $59(5)$ & $19(4)$ & 0.797 \\
\hline Cirrhosis (n) (\%) & $40(3)$ & $25(6)$ & 0.016 \\
\hline Cancer (n) (\%) & $97(7)$ & $42(9)$ & 0.194 \\
\hline Metastatic cancer (n) (\%) & $32(2)$ & $18(4)$ & 0.088 \\
\hline Renal disease $(n)(\%)$ & $243(18)$ & $142(31)$ & $<0.001$ \\
\hline CKD Stage $1(n)(\%)$ & 0 & 0 & NA \\
\hline CKD Stage $2(\mathrm{n})(\%)$ & $11(1)$ & $2(1)$ & 0.397 \\
\hline CKD Stage $3(n)(\%)$ & $51(4)$ & $36(8)$ & 0.001 \\
\hline CKD Stage $4(n)(\%)$ & $20(2)$ & $15(3)$ & 0.018 \\
\hline CKD Stage $5(n)(\%)$ & $2(1)$ & $2(1)$ & 0.263 \\
\hline End Stage Renal Disease (n) (\%) & $105(8)$ & $67(15)$ & $<0.001$ \\
\hline Acute Kidney Injury (n) (\%) & $265(20)$ & $111(24)$ & 0.050 \\
\hline $\operatorname{ICD}(\mathrm{n})(\%)$ & $37(3)$ & $12(3)$ & 0.856 \\
\hline Pacemaker (n) (\%) & $29(2)$ & $16(3)$ & 0.121 \\
\hline \multicolumn{4}{|l|}{ Heart Failure } \\
\hline $\operatorname{HFrEF}(n)(\%)$ & $88(7)$ & $40(9)$ & 0.141 \\
\hline $\operatorname{HFpEF}(n)(\%)$ & $120(9)$ & $80(17)$ & $<0.001$ \\
\hline \multicolumn{4}{|l|}{ Medications } \\
\hline Central Alpha Blocker (n) (\%) & $69(5)$ & $30(7)$ & 0.270 \\
\hline Alpha Blocker (n) (\%) & $41(3)$ & $9(2)$ & 0.211 \\
\hline Loop Diuretic (n) (\%) & $398(30)$ & $170(37)$ & 0.004 \\
\hline Thiazide Diuretic (n) (\%) & $115(9)$ & $37(8)$ & 0.709 \\
\hline Potassium sparing diuretic (n) (\%) & $133(10)$ & $51(11)$ & 0.488 \\
\hline Angiotensin Receptor Blocker (n) (\%) & $113(9)$ & $46(10)$ & 0.314 \\
\hline Dihydropyridine CCB (n) (\%) & $268(20)$ & $87(19)$ & 0.597 \\
\hline Non-Dihydropyridine CCB (n) (\%) & $75(6)$ & $30(7)$ & 0.473 \\
\hline Vasodilator (n) (\%) & $87(7)$ & $29(6)$ & 0.878 \\
\hline Beta Blocker (n) (\%) & $612(46)$ & $229(50)$ & 0.137 \\
\hline Angiotensin converting enzyme inhibitor (n) (\%) & $303(23)$ & $118(26)$ & 0.192 \\
\hline
\end{tabular}


1,781 discharged patients. The sample was separated into two groups. The first group comprised of patients who were not readmitted within 30 days and second group consisted of patients who were readmitted within 30 days of discharge. Population demographic and gender indicated no correlation. Of significance was the hospital readmissions in the prior year. The readmission group had an average of 1.61 hospital admissions in the prior year whereas the non-readmission group had an average of only 0.71 ( $P$ value $<0.001)$. In addition, the emergency department visits in the last 6-months also differed drastically between these two groups with 0.39 in the non-readmitted and 1.21 in readmitted group $(P$ value $<$ 0.001). Cumulative score indicators like HOSPITAL Score (4.96 in readmitted and 3.86 in non-readmitted group), LACE index (12.59 in readmitted and 11.31 in non-readmitted group), and Charlson Comorbidity Index (6.0 in readmitted and 4.88 in non-readmitted group) also indicated significantly higher values in readmitted group with $P$ values of each less than 0.001 in univariate analysis.

From the univariate analysis of medical comorbidities, the proportion of patients with HFpEF was observed to be significantly higher in the readmitted group compared to the non-readmitted group ( $17 \%$ vs. $9 \%, P$ value $<0.001)$, whereas the proportion of patients with HFrEF was not significantly different between the two groups $(9 \%$ vs $7 \%, P$ value $=$ 0.141 ). Renal disease was also observed as one of the significant factors affecting 30-day readmission with results of $18 \%$ in non-readmitted group and $31 \%$ in readmitted group $(P$ value $<0.01)$. When we further sub-grouped renal disease to acute kidney injury (AKI), chronic kidney disease (CKD stages I to V), and end stage renal disease (ESRD). We found that CKD stage 3 and 4, and ESRD had significant differences between the readmitted and non-readmitted group. The rates of prevalence of CKD III was $8 \%$ in readmitted and $4 \%$ nonreadmitted group $(P$ value $=0.001)$. Similarly, the rates of prevalence of CKD IV was 3\% in readmitted and $2 \%$ nonreadmitted group $(P$ value $=0.018)$. Further, ESRD was prevalent in $15 \%$ of readmitted group and $8 \%$ of nonreadmitted group ( $P$ value $<0.001$. The prevalence of acute kidney injury (AKI) were $24 \%$ in readmitted and $20 \%$ in non-readmitted with $P$ value of 0.050 . Further, we also found that $33 \%$ in readmitted group had history of myocardial infarction compared to $26 \%$ in the non-readmitted group ( $P$ value $=0.004)$. Similarly, $6 \%$ of patients in the readmitted group had cirrhosis compared to $3 \%$ in non-readmitted group $(P$ value $=0.016)$. Other comorbidities such as myocardial infarction and liver cirrhosis was $7 \%$ and $3 \%$ higher in readmitted vs. non-readmitted group with $P$ values of 0.004 and 0.016 respectively. We further looked into discharge medications as shown in the Table 1. Among these medications, loop diuretics showed increased association with readmission when compared with non-readmission group $(37 \%$ vs. $30 \%, P$ value $=0.004)$.

Figure 2 shows the results of multivariate analysis on significant variables from univariate analysis in the form of a forest plot of the odds ratio calculated from the analysis. We excluded parameters that were not significant in multivariate analysis from Figure 2. We found that odds ratio of HOSPITAL score 1.43 ( $P$ value $<0.001$ ); however, the odds ratio of LACE index was 0.87 with $P$ value of $<0.001$. For

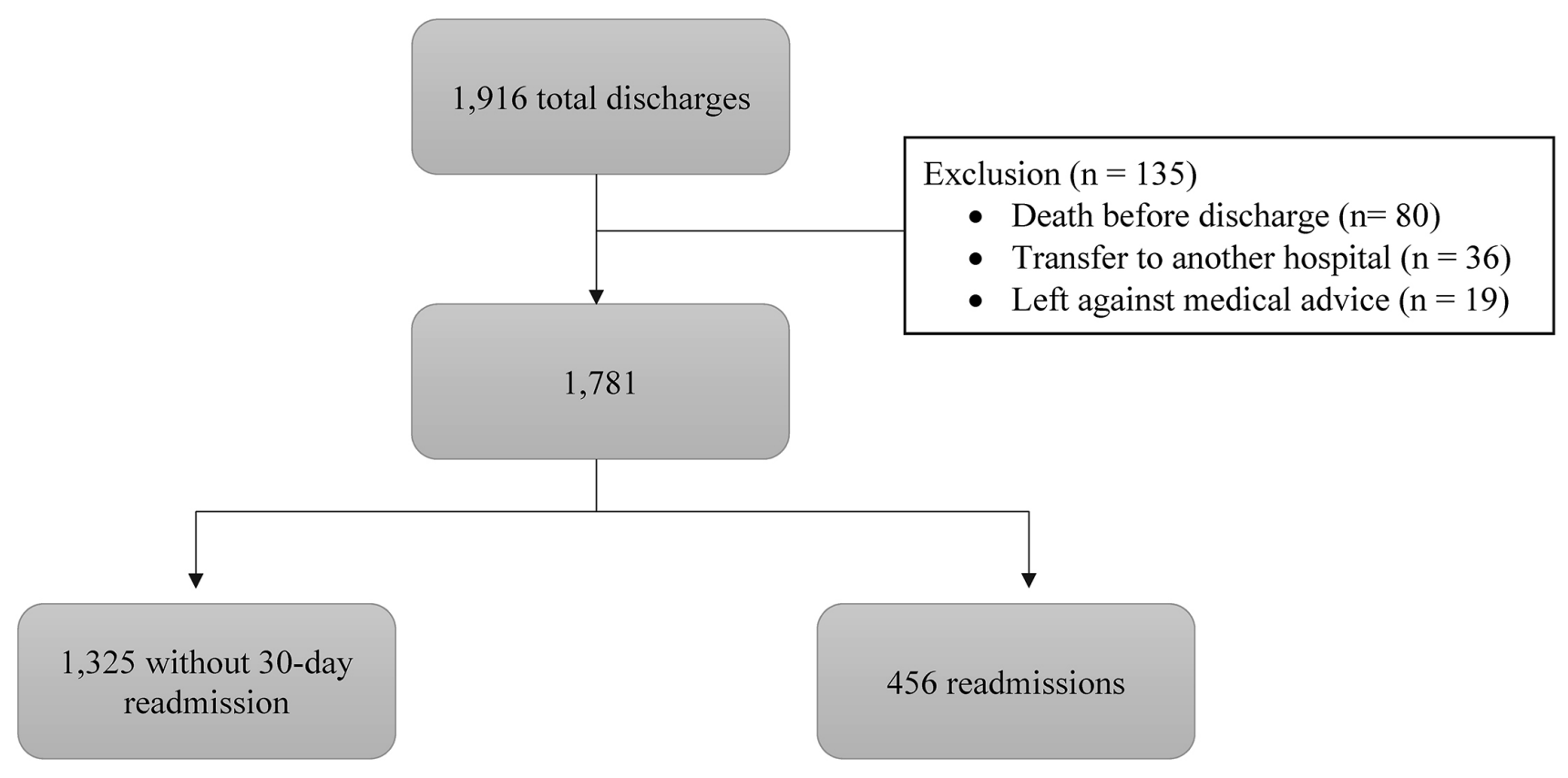

Figure 1. Flowsheet of total sample size 
HFpEF, odds ratio strengthened our results from univariate analysis with higher odds ratio of 1.77 (95\% CI: 1.25-2.50) with $P$ value 0.002 . Further, renal disease had odds ratio of 1.47 (95\% CI: $1.12-2.00)$ with $P$ value of 0.007 . Among the subgroup in renal disease, only CKD III was significant in the multivariate analysis with odds ratio of 1.74 (95\% CI: $1.05-$ 2.86) and $P$ value of 0.031 .

\section{Discussion}

Historically, studies have focused more on strategies to prevent heart failure. ${ }^{18}$ While these strategies discuss socioeconomic factors including financial burdens limiting access to resources and healthcare that contribute to readmissions in heart failure, they do not distinguish between the different types of heart failure. The data assessed in this study elucidates an important point that looks into heart failure and the differences in the readmissions risk, which differentiates our study from conventional heart failure studies.

Our study revealed that the proportion of patients with HFpEF was significantly higher in the readmitted group compared to the non-readmitted group $(17 \%$ vs. $9 \%, P<0.001)$. This higher likelihood could be due to a variety of causes. One of the causes being that HFpEF is more commonly seen in patients that have been diagnosed with significant co-morbidities such as severe lung disease, renal failure, diabetes and hypertension. ${ }^{19-23}$ Because the etiology of HFpEF may be multifactorial, management often requires a multifaceted approach. These cases cannot be handled solely in the inpatient setting, so it demands equal focus in the outpatient setting for optimal treatments and minimal hospitalizations. Unlike HFpEF, we did not find an association between HFrEF and 30-day readmissions. The proportion of patients with HFrEF was similar between the two groups $(9 \%$ vs $7 \%, P=0.141)$. A potential reason for this could be the established guidelines per ACA/AHA/HFSA for directed medical therapy on HFrEF targeted at the reduction of morbidity and mortality. ${ }^{23}$ The Guideline Directed Medical Therapy (GDMT) for HFrEF includes Angiotensin-Converting Enzyme Inhibitors (ACEI), or Angiotensin-Receptors Blockers (ARB) and Beta Blockers (BB), in all patients as well as Aldosterone Receptors Antagonists (ARA), nitrates and hydralazine in selected patients. The GDMT has shown to improve outcomes and increase survival in patients with HFrEF. With HFpEF, however, there are still ambiguities in evidence-based treatment regimens with no well-established GDMT. We can potentially assume that due to lack of specific evidence-based guidelines for HFpEF treatment, its effective management still remains as one of the challenges for health care providers.

Contradictory to our study, a retrospective study from 2014 , conducted in Boston, that compared 30-day readmissions between patients admitted with HFpEF and HFrEF showed that HFrEF have higher 30-days readmissions rate compared to $\mathrm{HFpEF}^{24}$ But, this was not seen after 30-days period. However, this study was included a different study setting compared to our study. It included only heart failure patients and the sample was from an urban population. While the findings of our study are definitely strong and insightful, it seems worthwhile to conduct large scale studies to identify if

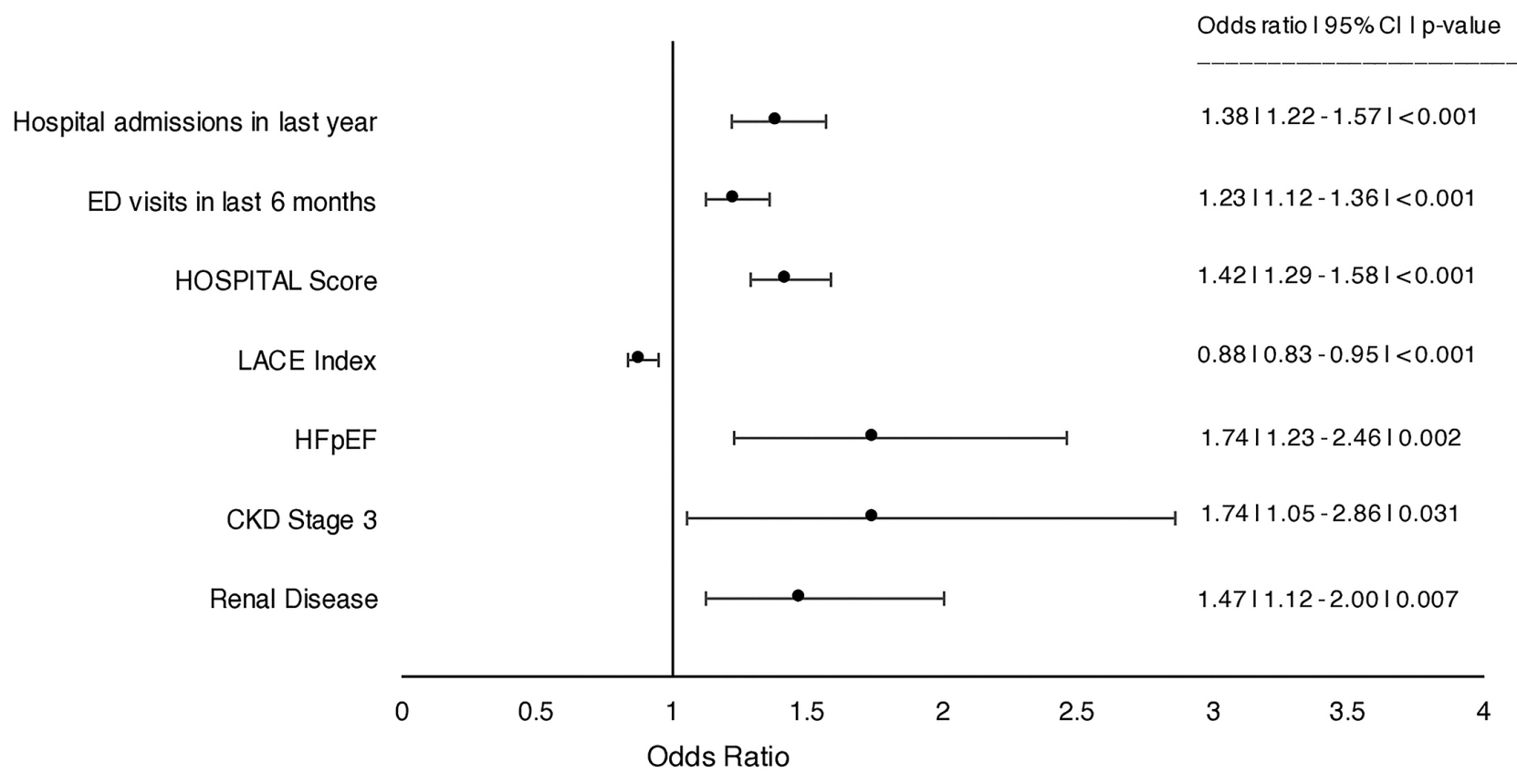

Figure 2. Forest Plot of multivariate analysis showing odds ratio 
there is any geographical or demographical factor that could influence the readmission rate.

We found that in our study population HOSPITAL score was effective in predicting readmissions showing higher values in both univariate as well as multivariate analysis. However, the LACE index that showed significantly higher values in readmitted population in univariate analysis failed to do so in multivariate analysis showing odds ratio of less than 1 . This is in an agreement with some prior studies that have highlighted that LACE index may not be an effective tool in predicting 30-day readmission. ${ }^{11,13-15}$

Renal disease was also observed as a notable risk factor that contributed to 30-day readmission risk. Renal disease, by itself, is an independent factor contributing for readmissions. ${ }^{25}$ Further, in patients with heart failure and co-existing renal failure, the risk becomes more pronounced. ${ }^{12}$ The spectrum of diseases that have association of heart diseases with renal diseases is called cardiorenal syndrome. There are different types of cardiorenal syndrome, including acute to chronic disease with complex pathophysiology behind each type. In inpatients, acute heart failure exacerbation is often associated with acute kidney injury due to difficulty in fluid balance. ${ }^{26}$ This difficulty in controlling fluid balance is also one of the potential reasons for the underlying association of heart failure and renal dysfunction with readmissions. Additionally, it has been noted through previous studies that heart failure, particularly on readmissions to the hospital, has been associated with decreased renal function. ${ }^{12}$ Complementary to these studies, our study found renal disease as a significant factor associated with 30-day readmissions. We also found that CKD stage 3 is independently associated with increased 30-day readmissions.

Myocardial infarction and liver cirrhosis are known co-morbidities that have impact on increasing 30-day readmission..$^{25,27}$ A study conducted to establish risk factors of readmissions showed that ischemic heart disease was not one of the top three medical co-morbidities for readmission and similar with liver disease. ${ }^{25}$ Our study showed initial association of increased risk of admissions in myocardial infarction and liver cirrhosis but failed to do so in multivariate analysis.

\section{Study Limitations}

The findings of this study are definitely insightful and add value to the existing knowledge about hospital readmissions. Nevertheless, there are some potential study limitations that need to be understood before generalizing or validating the findings of this study. First, the sample size was not large - the total eligible sample size of this study was 1781. Large sample studies may be needed to confidently assert the findings obtained here. Second, the current study focused on a single center of a mid-western city of the United States. This suggests the need for a wide range of geographical locations and multi-center study. Third, the eligible readmissions in this study included patients who were readmitted to the same hospital. This criterion could possibly have excluded some patients who were readmitted to another hospital, but we believe that those numbers would be a very tiny fraction since the hospital in the study is the only major hospital around the geographical area. Fourth, when computing HOSPITAL score, one of the components is discharge from oncology service. However, our department did not have a primary oncology service. Instead, we replaced that with patients who were discharged with a diagnosis for cancer. Future studies that could overcome these limitations would add more value and shed better confidence in the findings.

\section{Conclusion}

Our data supports HFpEF as an independent risk factor of readmissions in an academic tertiary care center. This association was found to be stronger than already validated HOSPITAL score and LACE index. However, no significant association was identified between HFrEF and 30-day hospital readmissions. This difference could possibly be because of the GDMT available for HFrEF and lack of evidence-based treatment available for HFpEF. Multidisciplinary management of HFpEF in both inpatient and outpatient settings may be an important target for interventions to reduce hospital readmissions.

\section{References}

1. Heart \& Vascular: Conditions \& Treatments. Heart Failure Statistics. Emory Healthcare Web site. Available at: https:// www.emoryhealthcare.org/heart-vascular/wellness/heartfailure-statistics.html. Accessed June 10, 2019

2. Heart Failure. Patient Education Handouts, Heart Disease Resources for Health Professionals. Centers for Disease Control and Prevention Web site. Available at: https://www. cdc.gov/dhdsp/data_statistics/fact_sheets/fs_heart_failure.htm. Accessed July 20, 2019.

3. Jencks SF, Williams MV, Coleman EA. Rehospitalizations among patients in the Medicare fee-for-service program. $\mathrm{N}$ Engl J Med. 2009;360(14):1418-1428.

4. Fernandez-Gasso L, Hernando-Arizaleta L, Palomar-Rodríguez JA, Abellán-Pérez MV, Pascual-Figal DA. Trends, causes and timing of 30-day readmissions after hospitalization for heart failure: 11-year population-based analysis with linked data. Int J Cardiol. 2017;248:246-251.

5. Ross JS, Chen J, Lin Z, et al. Recent national trends in readmission rates after heart failure hospitalization. Circ Heart Fail. 2010;3(1):97-103.

6. Kilgore M, Patel H, Kielhorn A, Maya J, Sharma P. Economic burden of hospitalizations of Medicare beneficiaries with heart failure. Risk Manag Healthc Policy. 2017;10:63-70.

7. Park C, Otobo E, Ullman J, et al. Impact on readmission reduction among heart failure patients using digital health monitoring: feasibility and adoptability study. JMIR Med Inform. 2019;7(4):e13353.

8. Burke RE, Schnipper JL, Williams MV, et al. The HOSPITAL Score predicts potentially preventable 30 -day readmissions in conditions targeted by the hospital readmissions reduction program. Med Care. 2017;55(3):285-290. 
9. Donzé JD, Williams MV, Robinson EJ, et al. International validity of the HOSPITAL score to predict 30-day potentially avoidable hospital readmissions. JAMA Intern Med. 2016;176(4):496-502.

10. van Walraven C, Dhalla IA, Bell C, et al. Derivation and validation of an index to predict early death or unplanned readmission after discharge from hospital to the community. CMAJ. 2010;182(6):551-557.

11. Damery S, Combes G. Evaluating the predictive strength of the LACE index in identifying patients at high risk of hospital readmission following an inpatient episode: a retrospective cohort study. BMJ Open. 2017 Jul 13;7(7):e016921.

12. Vindhyal MR, Khayyat S, Shaaban A, Duran BA, Kallail KJ. Decreased Renal Function is Associated with Heart Failure Readmissions. Cureus. 2018;10(8):e3122..

13. Robinson R, Hudali T. The HOSPITAL score and LACE index as predictors of 30 day readmission in a retrospective study at a university-affiliated community hospital. PeerJ. 2017;5:e3137.

14. Wang H, Robinson RD, Johnson C, et al. Using the LACE index to predict hospital readmissions in congestive heart failure patients. BMC Cardiovasc Disord. 2014;14:97.

15. Yazdan-Ashoori P, Lee SF, Ibrahim Q, Van Spall HGC. Utility of the LACE index at the bedside in predicting 30-day readmission or death in patients hospitalized with heart failure. Am Heart J. 2016;179:51-58.

16. Ibrahim AM, Koester C, Al-Akchar M, et al. HOSPITAL Score, LACE Index and LACE+ Index as predictors of 30-day readmission in patients with heart failure. BMJ Evid Based Med. 2020;25(5):166-167.

17. Goyal P, Loop M, Chen L, et al. Causes and temporal patterns of 30-day readmission among older adults hospitalized with heart failure with preserved or reduced ejection fraction. J Am Heart Assoc. 2018;7(9):e007785.

18. Ziaeian B, Fonarow GC. The prevention of hospital readmissions in heart failure. Prog Cardiovasc Dis. 2016;58(4):379-385.

19. Nanayakkara S, Patel HC, Kaye DM. Hospitalisation in patients with heart failure with preserved ejection fraction. Clin Med Insights Cardiol. 2018;12.

20. Hunt SA; American College of Cardiology; American Heart Association Task Force on Practice Guidelines (Writing Committee to Update the 2001 Guidelines for the Evaluation and Management of Heart Failure). ACC/AHA 2005 guideline update for the diagnosis and management of chronic heart failure in the adult - summary article: a report of the American College of Cardiology/American Heart Association Task Force on Practice Guidelines (Writing Committee to Update the 2001 Guidelines for the Evaluation and Management of Heart Failure). J Am Coll Cardiol. 2005;46(6):e1-e82.

21. Redfield MM, Jacobsen SJ, Burnett JC Jr, Mahoney DW, Bailey KR, Rodeheffer RJ. Burden of systolic and diastolic ventricular dysfunction in the community: appreciating the scope of the heart failure epidemic. JAMA. 2003;289(2):194202.

22. Cohn JN, Johnson G; Veterans Administration Cooperative Study Group. Heart failure with normal ejection fraction. The V-HeFT Study. Circulation. 1990;81(2)(Suppl):III48-III53.

23. Tran RH, Aldemerdash A, Chang P, et al. Guideline?directed medical therapy and survival following hospitalization in patients with heart failure. Pharmacotherapy: The Journal of Human Pharmacology and Drug Therapy. 2018;38(4):406-416.

24. Quiroz R, Doros G, Shaw P, Liang C, Gauthier DF, Sam F. Comparison of characteristics and outcomes of patients with heart failure preserved ejection fraction versus reduced left ventricular ejection fraction in an urban cohort. Am J Cardiol. 2014;113(4):691-696.

25. Donzé J, Lipsitz S, Bates DW, Schnipper JL. Causes and patterns of readmissions in patients with common comorbidities: retrospective cohort study. BMJ. 2013;347(dec16 4):f7171.

26. Rangaswami J, Bhalla V, Blair JEA, et al; American Heart Association Council on the Kidney in Cardiovascular Disease and Council on Clinical Cardiology. Cardiorenal syndrome: classification, pathophysiology, diagnosis, and treatment strategies: a scientific statement from the American Heart Association. Circulation. 2019;139(16):e840-e878.

27. Orman ES, Ghabril M, Emmett TW, Chalasani N. Hospital readmissions in patients with cirrhosis: a systematic review. J Hosp Med. 2018.

\section{Author Affiliations}

Manjari Rani Regmi, MD*; Mukul Bhattarai, MD, MPH, FACP*; Priyanka Parajuli, MD*; Odalys Estefania Lara Garcia, MD*; Nitin Tandan, MD*; Nicolas Ferry, MD, Capt USAFץ; Asad Cheema, MD*; Youssef Chami, MD*; Robert Robinson, MD, MS, FACP*

*Southern Illinois University School of Medicine, Springfield, Illinois, USA

†San Antonio Memorial Medical Center, San Antonio, Texas, USA 